\title{
Study of the Influence of Plaque Growth and Hydrostatic Properties in an Atherosclerotic Artery, for the Prevention of Arterial Wall Damage: Application to Vascular Diseases
}

\author{
J. R. Tsafack Nzifack $\mathbb{D}^{1},{ }^{1}$ J. S. Mabekou Takam ${ }^{D},{ }^{1}$ R. Moutou Pitti ${ }^{\circ},{ }^{2}$ M. Fogue, ${ }^{3}$ \\ and P. K. Talla $\left.{ }^{1}\right)^{1}$ \\ ${ }^{1}$ Unité de Recherche de Mécanique et de Modélisation des Systèmes Physiques (UR2MSP), \\ Université de Dschang, Dschang 237, Cameroon \\ ${ }^{2}$ Université de Clermont Ferrand, SIGMA Clermont, Institut Pascal, F63000, Clermont-Ferrand 33, France \\ ${ }^{3}$ Unité de Recherche d'Ingénierie des Systèmes Industriels et de l'Environnement (URSIE), IUT Fotso Victor de Bandjoun, \\ Bandjoun 237, Cameroon \\ Correspondence should be addressed to P. K. Talla; tpierrekisito@yahoo.com
}

Received 27 October 2020; Accepted 24 April 2021; Published 10 May 2021

Academic Editor: Jean-Michel Bergheau

Copyright $\odot 2021$ J. R. Tsafack Nzifack et al. This is an open access article distributed under the Creative Commons Attribution License, which permits unrestricted use, distribution, and reproduction in any medium, provided the original work is properly cited.

\begin{abstract}
The objective of this work is to prevent damage of the arterial wall, using a theoretical model of hyperelastic, anisotropic, and dynamic behavior of the human arterial. This work is mainly focused on the properties of the hydrostatic stress and the evolution of stenosis. This work is mainly focused on the properties of the hydrostatic stress and the evolution of stenosis in order to understand the effect of the size of the plaque deposit, the loss of elasticity of the wall, and the increase in the density of the blood on the mechanical behavior of the human arterial wall. The great contribution of this work shows us that increasing the size of the plaque also increases arterial stress, and the radial growth of the plaque is very dangerous compared to the longitudinal growth. Furthermore, atherosclerosis promotes the loss of elasticity of the arterial wall and increases the density of blood mass. Indeed, all these subsequent phenomena increase arterial stress. All the results are in good agreement with the expected result of the literature and could play an important role in the diagnosis of the patient with an arterial injury. It will also help the doctor and the surgeon to make a good clinical decision and good treatment planning.
\end{abstract}

\section{Introduction}

Health problems are a great concern for people today [1]. For centuries, cardiovascular disease has been identified as one of the major diseases affecting many people [2]. Stenosis or arteriosclerosis is the abnormal and unnatural growth of the thickness of the arterial wall, which develops at various locations in the cardiovascular system under conditions of pathology [3, 4]. This may be due to unhealthy living conditions such as exposure to tobacco smoke, lack of physical activity, and poor eating habits. Carotid stenosis artery is a narrowing of the large arteries that carry blood to the head, face, and brain [5]. The narrowing is usually the result of an accumulation of plaque in the arteries (atherosclerosis). The stenosis can worsen over time and completely block the artery, which can lead to a stroke [5]. Thus, it leads to the modification of blood pressure, causing flow disorders and increased hemodynamic stress of the arterial wall, which will damage the endothelium [6]. It is therefore important to study the hemodynamic stress of the artery in relation to the modification (of the density of the blood, the lumen of the artery, and the elasticity of the wall) and to understand the evolution of the parameter that is associated with arterial pathology. For this, we carried out the modelling of the hemodynamic stress of the elastic artery, with variation of the degree of stenosis, of the density of the blood, and of the elasticity of the wall. Yet in the clinical field, doctors and surgeons determined the arterial narrowing by simply measuring the height of the stenosis [7]. This is insufficient for clinical decision-making and 
treatment planning because it is not the only geometric parameter affecting the stress of the arterial wall. Thus, the anomalies of the deformation of the arteries due to narrowing, dilation, and properties of materials such as the elasticity of the wall and the mass density of the blood should be considered. Indeed, the latter allows us to assess the parameters of hemodynamic stress more realistically, and this in turn allows doctors to better understand arterial disease and the consequences of the progression of plaque growth, the modification of the mass density of blood, and elasticity of the wall. To achieve this, we will focus our view on certain works in the literature on the mechanical behaviour of the arterial wall. For example, reference [8] has shown the involvement of the disturbance of the metabolism of smooth muscle cells and the degradation of collagen in the medial layer with the appearance of aneurysm and arterial dissection. To understand the tear phase of an aortic dissection, reference [9] studied the response of the thoracic aorta due to radial and longitudinal forces. The acquisition of the modification of the mechanical properties of the different arterial layers in vivo is undertaken in reference [10] to demonstrate the recovery of the wall during a pathological manifestation. The evaluation of Von-Mises stress was investigated in reference [11] to show that the maximum stress is at the media level and to validate its numerical simulations with the results obtained by medical techniques. For the aneurysm rupture study, references $[12,13]$ used hemodynamic, morphological, and mechanical parameters. The variation of viscosity of the fluid was considered by reference [14] to study the shear stresses generated on the wall of the aneurysm. Reference [15] studied health problems that have apparently concerned people today [1]. Stenosis or arteriosclerosis is the abnormal and unnatural growth of the thickness of the arterial wall which develops at various places in the cardiovascular system under conditions of pathology $[3,4]$. This may be due to unhealthy living conditions such as exposure to tobacco smoke, lack of physical activity, and poor eating habits. Carotid stenosis artery is a narrowing of the large arteries that carry blood to the head, face, and brain [5]. The narrowing is usually the result of an accumulation of plaque in the arteries: atherosclerosis. The stenosis can worsen over time and completely block the artery, which can lead to a stroke [5]. Thus, it leads to the modification of blood pressure, causing flow disorders and increased hemodynamic stress of the arterial wall, which will damage the endothelium [6]. It is therefore important to study the hemodynamic stress of the artery in relation to the modification (of the density of the blood, the lumen of the artery, and the elasticity of the wall) and to understand the evolution of the parameter that is associated with arterial pathology. For this, we carried out the modelling of the hemodynamic stress of the elastic artery, with variation of the degree of stenosis, of the density of the blood, and of the elasticity of the wall. Yet in the clinical field, doctors and surgeons determined the arterial narrowing by simply measuring the height of the stenosis [7]. This is insufficient for clinical decision-making and treatment planning because it is not the only geometric parameter affecting the stress of the arterial wall. Thus, the anomalies of the deformation of the arteries due to narrowing, dilation, and properties of materials such as the elasticity of the wall and the mass density of the blood should be considered. Indeed, the latter allows us to assess the parameters of hemodynamic stress more realistically, and this in turn allows doctors to better understand arterial disease and the consequences of the progression of plaque growth, the modification of the mass density of blood, and elasticity of the wall. To achieve this, we will focus our view on certain works in the literature on the mechanical behaviour of the arterial wall. For example, reference [8] has shown the involvement of the disturbance of the metabolism of smooth muscle cells and the degradation of collagen in the medial layer with the appearance of aneurysm and arterial dissection. To understand the tear phase of an aortic dissection, reference [9] studied the response of the thoracic aorta due to radial and longitudinal forces. The acquisition of the modification of the mechanical properties of the different arterial layers in vivo is undertaken by reference [10] to demonstrate the recovery of the wall during a pathological manifestation. The evaluation of Von-Mises constraints was investigated by reference [11] to show that the maximum stress is at the media level and to validate its numerical simulations with the results obtained by medical techniques. For the aneurysm rupture study, references [12, 13] used hemodynamic, morphological and mechanical parameters. Reference [15] used photoelasticimetry and numerical studies to assess the wall stresses of the aneurysm. The results that they obtained are validated by medical techniques, but do not take into account the stress of the wall, linked to the modification of the mass density of blood, arterial light, and the elasticity of the wall. Thus, within the framework of this study, we are interested in the influence of the degradation of the wall due to the growth of the plaque and to the modification of the density of the blood and the rigidity of the arterial wall. Using the theoretical model of hyperelastic, anisotropic, and dynamic mechanical behaviours of the human common carotid artery, considering the growth of plaque and hydrostatic property, we analyse the plaque growth elasticity and the density of the blood and numerical studies to assess the wall stresses of the aneurysm. The results that they obtained are validated by medical techniques, but do not take into account the stress of the wall, linked to the modification of the mass density of blood, arterial light, and the elasticity of the wall. Thus, within the framework of this study, we are interested in the influence of the degradation of the wall due to the growth of the plaque and to the modification of the density of the blood and the rigidity of the arterial wall. Using the theoretical model of hyperelastic, anisotropic, and dynamic mechanical behaviours of the human common carotid artery, considering the growth of plaque and hydrostatic property, we analyse the plaque growth elasticity and the density of the blood.

\section{Theoretical Modeling of the Mechanical Behaviour of the Arterial Wall}

This article is concerned with the mathematical modelling of the effects of deposit plaque on the mechanical properties of the soft biological tissues that constitute the walls of arteries. Many important aspects of the mechanical behaviour of 
arterial tissue can be treated on the basis of elasticity theory. The focus of the article is therefore on the constitutive modelling of the anisotropies and highly nonlinear elastic properties of the atherosclerosis artery wall. The fundamental features of the relevant theory are summarized briefly, and particular forms of the elastic strain-energy function are discussed and then applied to an artery considered as a thick wall, a circular cylindrical tube in order to illustrate its extension inflation behaviour. The wide range of applications of the constitutive modelling framework to artery walls in both health and disease and to the other fibrous soft tissues is discussed in detail.

2.1. Kinematics. The kinematics of the structure can be described by two successive transformations in a system of cylindrical coordinates relative to the base $\left(e_{r}, e_{\theta}, e_{z}\right)$; the deformation is measured by the gradient of the $F$ transformation [16]:

$$
F=\left[\begin{array}{ccc}
\lambda_{r} & 0 & 0 \\
0 & \lambda_{\theta} & 0 \\
0 & 0 & \lambda_{z}
\end{array}\right],
$$

where $\lambda_{r}, \lambda_{\theta}$, and $\lambda_{z}$ are the main elongations in the radial, circumferential, and axial directions, respectively. The right and left Cauchy-green expansion tensor is given by $C=F^{t} F$ and $B=F F^{t}$ and $F^{t}$ represents the transpose tensor $F$.

2.2. Anisotropic Behavioural Relationship. The arterial wall is essentially composed of water, it is generally considered an incompressible medium [17], and the fluid movement inside the porous tissue induces stress gradients. This hypothesis of wall incompressibility has been demonstrated by experimental studies. Reference [18] has shown that artery samples deform in isochoric ways without the action of a large number of different loads.

In this work, we have exploited the deformation energy of Holzapfel, proposed in 2005. This energy takes into account the radial dispersion of fiber distribution in the arterial wall and has the following expressions:

$$
\begin{aligned}
W= & \left(I_{1}, I_{4}\right)=\frac{\mu}{2}\left(I_{1}-3\right) \\
& +\frac{k_{1}}{k_{2}}\left[\exp k_{2}\left((1-\rho)\left(I_{1}-3\right)^{2}+\rho\left(I_{1}-3\right)^{2}\right)\right]-1,
\end{aligned}
$$

where $\rho \in[0,1]$ is a dispersion factor that regulates the degree of anisotropy [19]. In an original way, if $\rho=0$, we obtain the composition of a neo-Hookean model with the [20] model, and if $\rho=1$, we obtain the [17] model. The [21] model has been used by several authors to study physiological stress by different arterial segments. Reference [22] uses this model to simulate coronary surgery and also by [23] to model the behaviour of healthy arteries. Finally, this model will be the most appropriate in our current study.
2.3. Stress. Cauchy's stress can be broken down into a passive contribution and an active contribution [16].

2.3.1. Passive Contribution. The Cauchy tensor is given by

$$
\sigma=\frac{2}{J} F \frac{\partial W\left(I_{1}, I_{4}\right)}{\partial c} F^{t},
$$

with

$$
\begin{aligned}
& I_{1}=\lambda_{r}^{2}+\lambda_{\theta}^{2}+\lambda_{z}^{2} ; \\
& I_{4}=\lambda_{\theta}^{2} \cos (\varphi)+\lambda_{z}^{2} \sin (\varphi) .
\end{aligned}
$$

For an incompressible material $J=\operatorname{det} F$, the work of [18] has shown that the arterial wall is incompressible and the Cauchy stress becomes $\sigma=-\Delta P I+(2 / J) F\left(\partial W\left(I_{1}, I_{4}\right) / \partial c\right) F^{t}$, where $\Delta P$ represents a hydrostatic stress contribution. Let us now write this expression in the form of [24]

$$
\begin{aligned}
\sigma= & -\Delta p I+2 W_{1} B+2 W_{2}\left(I_{1} 1-B\right) B+2 W_{4} a \otimes a \\
& +2 W_{5}(a \otimes B a+B a \otimes a),
\end{aligned}
$$

where $\otimes$ represents the tensor product.

With $W_{j}=\left(\partial W / \partial I_{j}\right)(j=1,2,3,4,5)$. It should also be noted that an energy function that characterizes a material must respect the principle of material indifference, also called the principle of objectivity. It must be independent of the reference system. Other mathematical criteria must normally be met, such as convexity and ellipticity.

(1) Modeling of Hydrostatic Stress. In continuum mechanics, a hydrostatic stress is anisotropy stress that is given by the movement of blood inside the artery. It is often used interchangeably with pressure and is also known as confining stress. Its magnitude can be given by

$$
\Delta P=R_{p} Q \text {, }
$$

where $R_{p}$ is the resistance and $Q$ the blood flow. This relationship is only valid for a perfectly developed and symmetric axial flow. The resistance explains the viscous loss along the pipe and is calculated as follows:

$$
R_{p}=\frac{8 \mu L}{\pi r^{4}}
$$

where $\mu$ is the dynamic viscosity of the blood and Land $r$ are the length and radius of the vessel, respectively. Due to the fact that the resistance is proportional to the radius, a small change or a great change of the radius will modify the resistance. This can be caused by the presence of the stenosis in the lumen of the artery.

(2) Modeling of Stenosis. Fat accumulation, observed in the arterial stenosis, forms a rough and uneven surface, thus decreasing the effective diameter of the vessel. In a $0 D$ representation of the vessel, a stenosis can be modeled with ad-hoc resistance. To calculate the resistance, the vessel is divided into two segments, one part stenosis and the other part steroid. For simplicity, the radius lumen in the stenosis 
portion is assumed to be a constant value along the stenosis. Figure 1 shows the idealized geometry of a stenosis.

The effective resistance of the vessel stenosis can be calculated as follows:

$$
R_{p}=\frac{8 \mu}{\pi r_{0}^{4}}\left(l_{0}-l_{s}\right)+\frac{8 \mu}{\pi r_{s}^{4}} l_{s},
$$

where $r_{0}, l_{0}$ represent the resistance and length of the healthy part of the vessel. $r_{s}, l_{s}$ denote the resistance and length of the stenosis part of the vessel.

Since our aim is to study the dynamic of blood flow of the cerebral circulation, it will be advantageous to fix our eyes on the Fahraors-Lindqvist effect, which will allow us to express the resistance above according to the discharge hematocrit $H_{D}$ and the vessel diameter $D$, defining the $\mu$ viscosity in vivo as

$$
\begin{aligned}
\mu= & \mu_{p}\left[1+\left(\mu_{1}^{0.45}-1\right) \frac{\left(1-H_{D}^{c}\right)-1}{(1-0.45)^{c}-1} *\left(\frac{D}{D-1.1}\right)^{2}\right] \\
& *\left(\frac{D}{D-1.1}\right)^{2},
\end{aligned}
$$

with $\mu_{p}$ the viscosity of the initial plasma $9^{*} 10^{-6} \mathrm{mmHgS}$ and $\mu_{1}^{0.45}$ the viscosity of the blood for a hematocrit flow of 0.45 , which is given by the expression

$$
\begin{aligned}
\mu_{1}^{0.45}= & 6 \exp (-0.085 D)+3.2-2.44 \exp \left(-0.006 D^{0.0645}\right) \\
C= & (0.8+\exp (-0.075 D)) *\left(-1+\frac{1}{1+10^{-11} D^{12}}\right) \\
& +\frac{1}{1+10^{-11} D^{12}} .
\end{aligned}
$$

\section{(3) Artery Brain Posterior.}

Model definition

The modified Lumped parameter model [26] is to study $\mathrm{CBF}$ regulation. A lumped parameter model was developed (Figure 2), describing the segment of the posterior cerebral artery (PCA) and its distal vessels, up to the large cerebral veins. This segment was chosen since it supplies the primary visual cortex with blood [27]. The model consists of three lumped parts: the PCA, the arteriolar circulation and microcirculation, and the venous circulation.

The vessel compliance $\left(C_{p}\right)$

The PCA is modeled by a line model. Full PCA compliance $\left(C_{p}\right)$ is considered using a capacitor

$$
C_{p}=\frac{3 \pi r_{p}^{2}\left(\left(r_{p} / h_{p}\right)+1\right)^{2}}{E_{p}\left(\left(r_{p} / h_{p}\right)+1\right)} l_{p},
$$

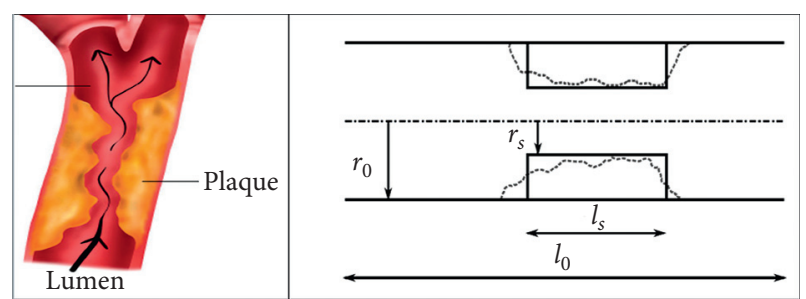

FIGURE 1: Sketch of arterial vessel segment with stenosis and 0Dmodel [25].

where $r_{p}, E_{p}, h_{p}$, and $l_{p}$ are in respect artery inner radius, Elasticity modulus, thickness of artery and PCA length.

For the reason of this simulation, it should be noted that, by passing blood thorough vessels, the vessels would be expanded or contracted. So, they can keep blood or release it and this is exactly like what a capacitor does.

The blood inertia $\left(L_{p}\right)$

The blood inertia $\left(L_{p}\right)$ is simulated by inductors:

$$
L_{p}=\frac{\rho_{b}}{\pi r_{p}^{2}} l_{P},
$$

where $\rho_{b}, l_{P}$, and $r_{p}$ are, respectively, blood density, PCA length, and artery inner radius.

The reason for this consideration is the variability of flow acceleration in pulsatile blood flow. So, an inductor can model inertia of blood flow very clearly.

(4) State Equations of Lumped Parameter Model. The states equations of lumped parameter and its parameters are given in this section

\section{Artery brain posterior}

The PCA model involves three equations of states:

$$
\begin{aligned}
& \frac{\mathrm{d} P_{a i}}{\mathrm{~d} t}=\frac{2\left(Q_{a i}-Q_{l p}\right)}{C_{p}}, \\
& \frac{\mathrm{d} Q_{l p}}{\mathrm{~d} t}=\frac{P_{a i}-P_{p}-Q_{l p} R_{p}}{L_{p}}, \\
& \frac{\mathrm{d} P_{p}}{\mathrm{~d} t}=\frac{2\left(Q_{l p} R_{a}-P_{p}+2 P_{a}\right)}{R_{a} C_{p}},
\end{aligned}
$$

where $P_{a i}, Q_{a i}, Q_{l p} C_{p}, P_{p}, R_{p}, R_{a}$, and $P_{a}$ are respect arterial input, PCA inflow, inertia flow, compliance, PCA output pressure, PCA resistance, arteriolar resistance, and central arteriolar.

The passive becomes when $\Delta p=P_{a i}$ before stenoses and et $\Delta p=P_{p}$ after stenoses 


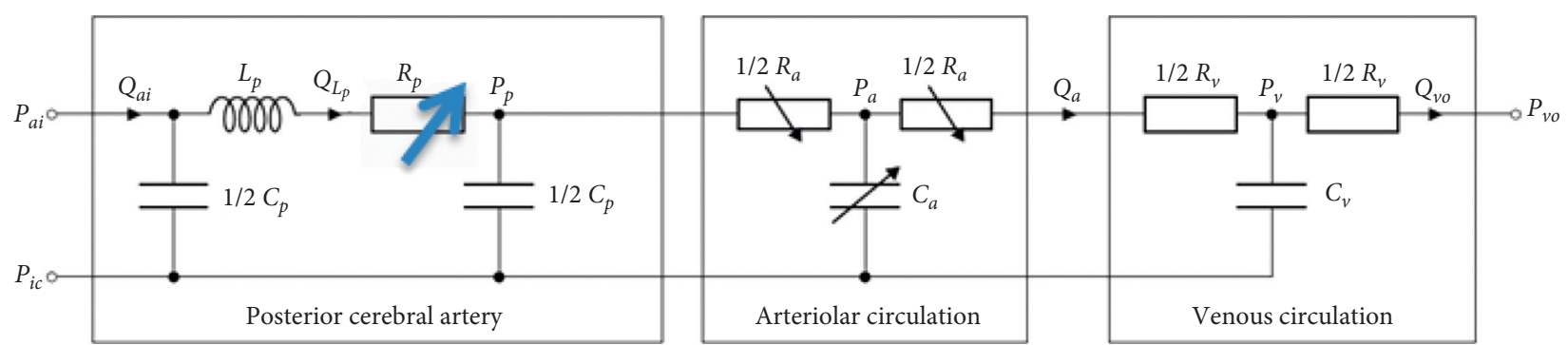

Figure 2: Lumped parameter model of the posterior cerebral artery (PCA) and its distal arteriolar and venous beds. $P_{a i}, P_{i c}$, and $P_{v 0}$ : arterial entry, intracranial, and venous exit blood pressure. $P_{P}$ : output pressure of the PCA. $P_{a}$ and $P_{v}$ : central and venous blood pressure. Qai and QLp: PCA input and inertia flow. $Q_{a}$ and $Q_{v 0}$ : venous entry and exit streams. $C_{p}, L_{p}$, and $R_{p}$ : PCA compliance, inertia, and resistance, respectively. $c_{a}$ and $R_{a}$ : arteriolar compliance and resistance. $c_{v}$ and $R_{v}$ : venous compliance and resistance. Posterior cerebral [26].

$$
\begin{aligned}
\sigma= & -P_{a i} I+2 W_{1} B+2 W_{2}\left(I_{1} 1-B\right) B+2 W_{4} a \otimes a \\
& +2 W_{5}(a \otimes B a+B a \otimes a), \\
\sigma= & -P_{p} I+2 W_{1} B+2 W_{2}\left(I_{1} 1-B\right) B+2 W_{4} a \otimes a \\
& +2 W_{5}(a \otimes B a+B a \otimes a) .
\end{aligned}
$$

2.3.2. Active Stress. The first active stress models were developed in the context of small deformation [28] and then extended in the context of large deformation [29-31]. Thus, the mode of [32] allows to analyze the dynamic behavior of the arterial wall. This model has for expression:

$$
\sigma_{\text {act }}=\lambda_{i} T_{0}\left[C_{a}^{2+}\right]\left(1-\left(\frac{\lambda_{m}-\lambda_{i}}{\lambda_{m}-\lambda_{0}}\right)^{2}\right)\left(e_{i} \otimes e_{i}\right),
$$

where $\otimes$ is the tensor product, $\lambda_{i}$ is the elongation in the direction of the smooth muscle cells, and $\lambda_{m}$ is the elongation by which the stress is maximum and $\lambda_{0}$ the one for which it stops $T_{0}\left[C_{a}^{2+}\right]$ and the activation function is function of calcium. his model was modified by reference [33] by taking the activation function in the form of:

$$
T_{0}\left[C_{a}^{2+}\right]=T_{m}\left[1-\exp \left(-C^{2}\right)\right], \quad \text { with } C=C_{B}-C_{S}\left(\frac{\tau_{w}-\tau_{w}^{h}}{\tau_{w}^{h}}\right),
$$

where $T_{m}$ is the maximum tension generated by a contraction, $C_{B}$ the basal value of the vasoconstrictor, and $C_{S}$ allows the regulation of vasomotor activity by endothelial cells receiving shear stresses due to blood flow $\tau_{w}$, compared to a homeostatic value $\tau_{w}^{h}$.

The total stress will be the sum of passive, and active stress according to the expression:

$$
\sigma=\sigma^{p}+\sigma_{\text {act }},
$$

$\sigma^{p}$ is given by the relationship (5). $\sigma_{\text {act }}$ is given by the relationship (15).

\subsection{Parameters Determination}

2.4.1. Active Stress. With regard to the active stress, we will use the parameters of [33] which are $T_{m}=150 \mathrm{kPa} ; \lambda_{m}=$ $1.1 ; \lambda_{0}=0.4 ; C_{b}=0.68 ; C_{s}=20 C_{B}$.
2.4.2. Hyperelastic Model Parameters. These parameters given by [21] are $\mu=2.54 \mathrm{kPa} ; k_{1}=21.6 \mathrm{kPa} ; k_{2}=8.28[-]$; and $\rho=0.25[-]$

2.4.3. Lumped Parameters Model. The constant lumped parameters model details is presented in Table 1.

\section{Results and Discussion}

3.1. Study of the Influence of Atherosclerosis on the Arterial Wall Resistance. Figures 3-5 show the influence of the growth of atherosclerosis on the resistance of the arterial wall. The results show that when the degree of stenosis increases, the stress on the wall also increases. These results are in agreement with literature [38]. This can be explained by the fact that the change in radius, leads to a significant change in wall resistance because the lumen become small and the platelet's activation are high [39]. In fact, when the lumen is gradually blocked, the lumen becomes small. This phenomenon has physiological consequences because it alters the transport of oxygen and other essential metabolites and may even trigger a pathological disorder. How is this possible?

The atheroma plaque, nourished by fats circulating in the blood, gradually develops on the wall of the artery while causing a narrowing (stenosis) hampering the passage of blood and the supply of oxygen to the organ that it irrigates [40]. It also constitutes a real clot trap [41]. When passing through the stenosis, the blood flow slows down, tending to deposit clotting elements (platelet and fibrin) there. This leads to the formation of stenosis, which decreases blood flow and compromises the oxygenation of the tissues supplied by the diseased artery. The installation of progressive stenosis can increase the wall stress [42] which will cause cerebral ischaemia or even haemorrhage.

This is why our result shows us an increase of stress as the stenosis increases. Why fixing the degree of longitudinal stenosis at $10 \%$ of the initial length, and we radially varied the height of the plaque $(33.33 \% ; 50 \%$; and $83 \%$ reduction of the arterial lumen), this presents us the evolution of the stress.

3.2. Comparative Study of the Deposition of Plaque. The aim here was to assess the stress of the arterial wall as a function 
TABle 1: Constant lumped parameters model symbol, value, unit, description, and reference.

\begin{tabular}{|c|c|c|c|c|}
\hline Symbol & Value & Unit & Description & Reference \\
\hline$\rho_{b}$ & 1.05 & $\mathrm{~g} / \mathrm{ml}$ & Blood density & [34] \\
\hline$E_{p}$ & 1.6 & $\mathrm{MPa}$ & Elasticity modulus of PCA wall & [35] \\
\hline$P_{\nu 0}^{P}$ & 14 & $\mathrm{mmHg}$ & Venous output pressure & [36] \\
\hline$P_{i c}$ & 10 & $\mathrm{mmHg}$ & Intracranial pressure & [35] \\
\hline$Q_{b l}$ & 53 & $\mathrm{ml} / \mathrm{min}$ & Baseline flow & [37] \\
\hline$\eta_{a}$ & 47.8 & $\mathrm{mmHg}$ & Wall viscosity & {$[36]$} \\
\hline$R_{p}$ & 0.105 & $\mathrm{~cm}$ & PCA inner radius & {$[34,35]$} \\
\hline$L_{p}^{p}$ & 8.6 & $\mathrm{~cm}$ & PCA length & Mulder et al. [35] \\
\hline$h_{p}^{p}$ & 0.026 & $\mathrm{~cm}$ & PCA thickness & {$[35]$} \\
\hline$r_{a 0}^{P}$ & 0.0075 & $\mathrm{~cm}$ & Vessel inner radius in the condition of unstressed wall & [36] \\
\hline$h_{a 0}$ & 0.0025 & $\mathrm{~cm}$ & Wall thickness in the unstressed condition $r \mathrm{a} 0$ & [36] \\
\hline
\end{tabular}

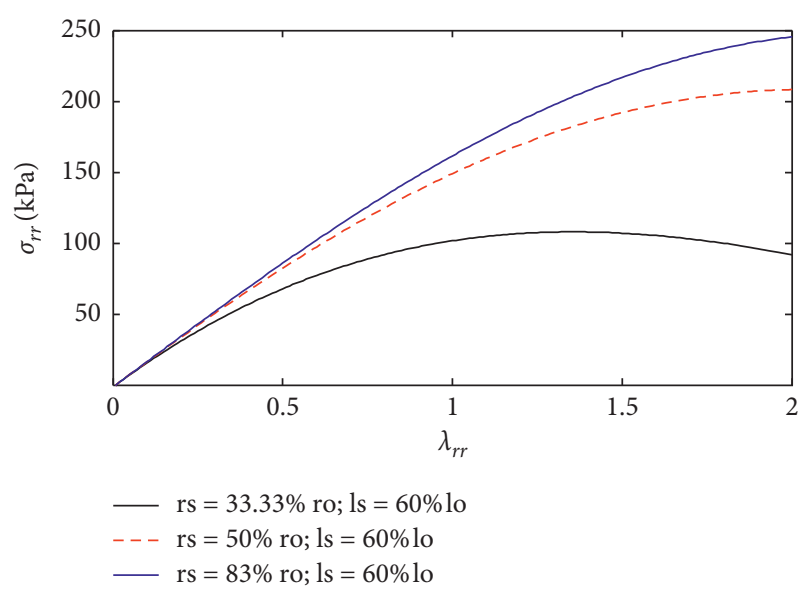

FIGURE 3: Study of the influence of stenosis on radial stress.

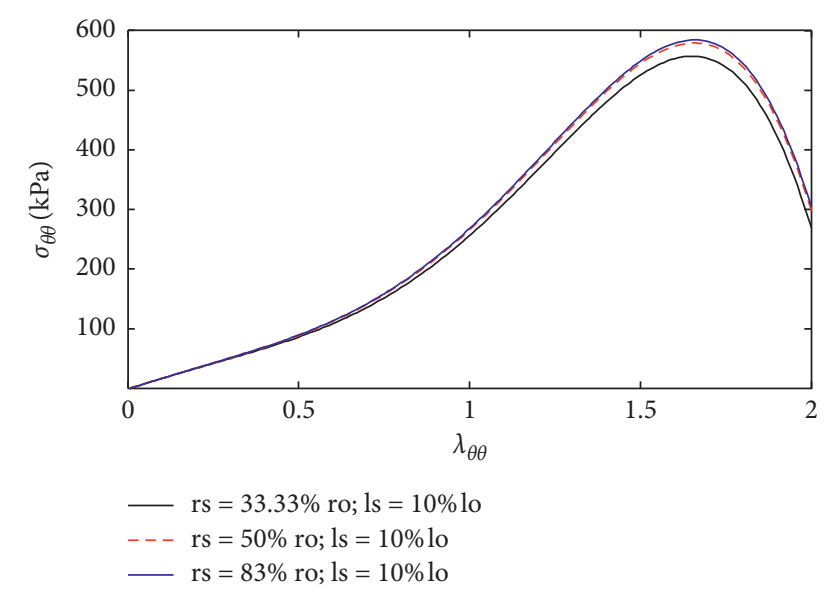

FIGURE 4: Study of the influence of the stenosis on the circumferential stress.

of the percentage of longitudinal and radial reduction. Figures 6-8 present the situation. It is clear that when the percentage reduction in the longitudinal section of the stenosis is less than the percentage in the radial section, the stress is higher, and if not, the stress is less high. Then, when the latter is equal, the greater equality has the higher stress than the average equality. The detailed remarks of these

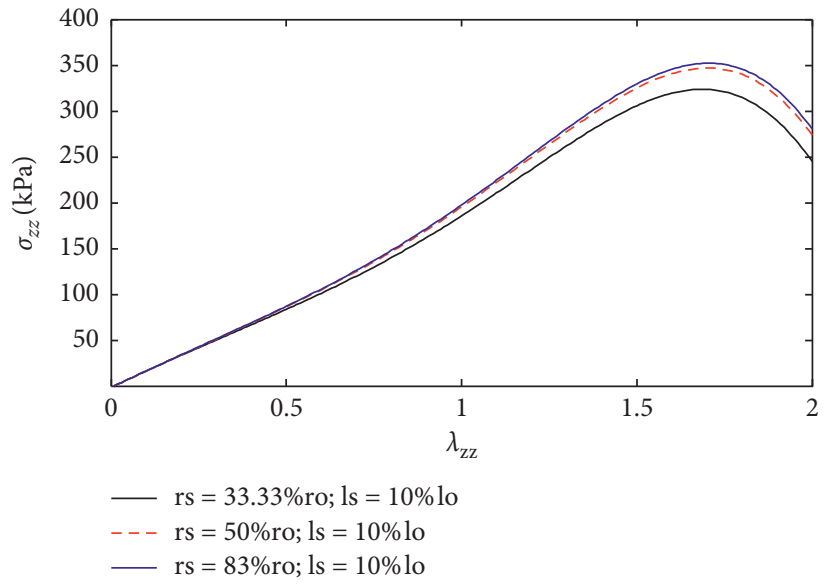

Figure 5: Study of the influence of stenosis on axial stress.

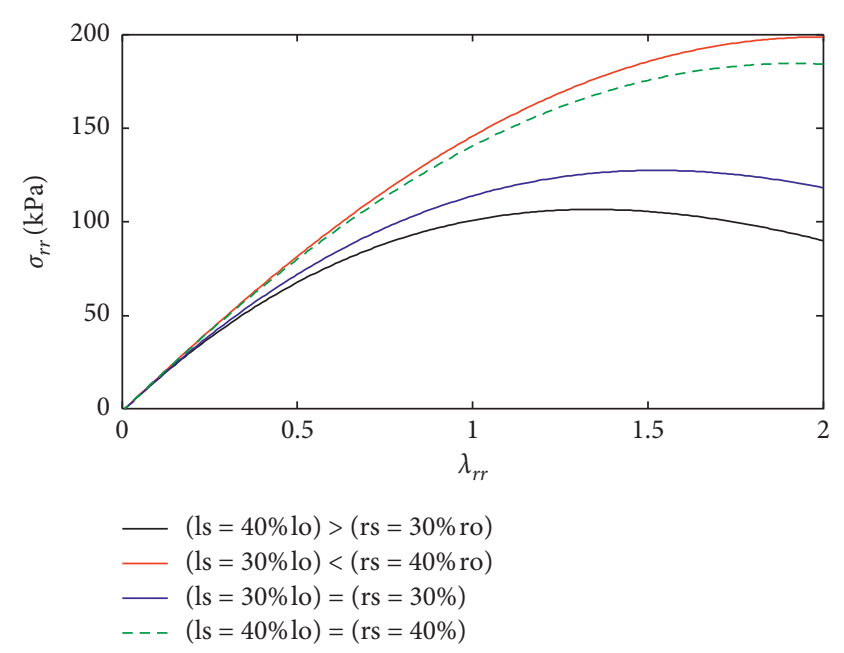

Figure 6: Comparative study of the deposition of plaque on radial stress.

Figures 6-8 show us that the percentage of radial reduction is more dangerous than the percentage of longitudinal reduction, which is an agreement with the result of the literature [39]. 


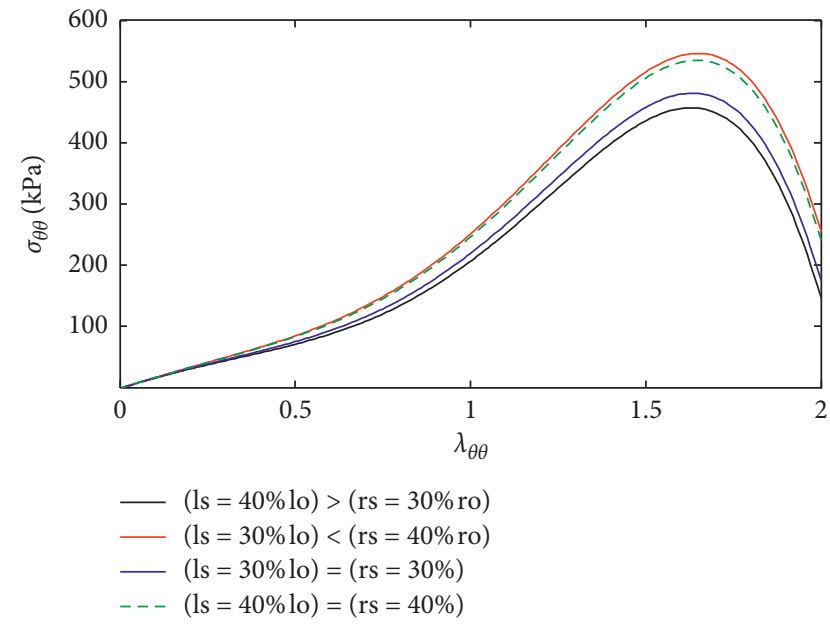

Figure 7: Comparative study of the deposition of plaque on the circumferential stress.

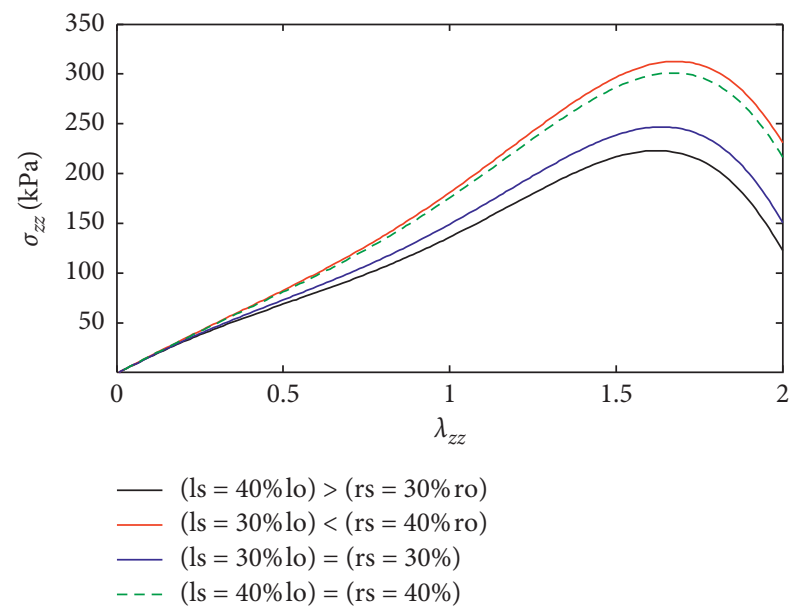

Figure 8: Comparative study of the deposition of plaque on the axial stress.

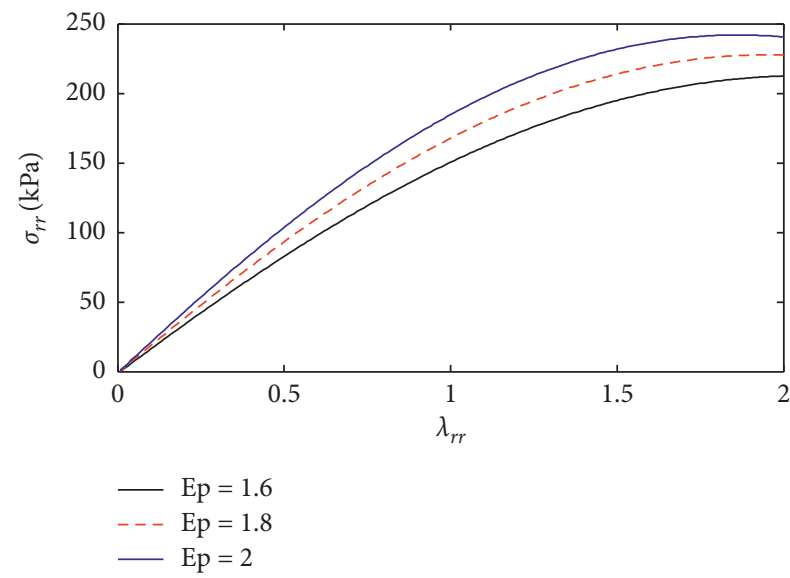

Figure 9: Study of the influence of the elasticity modulus on radial stress.

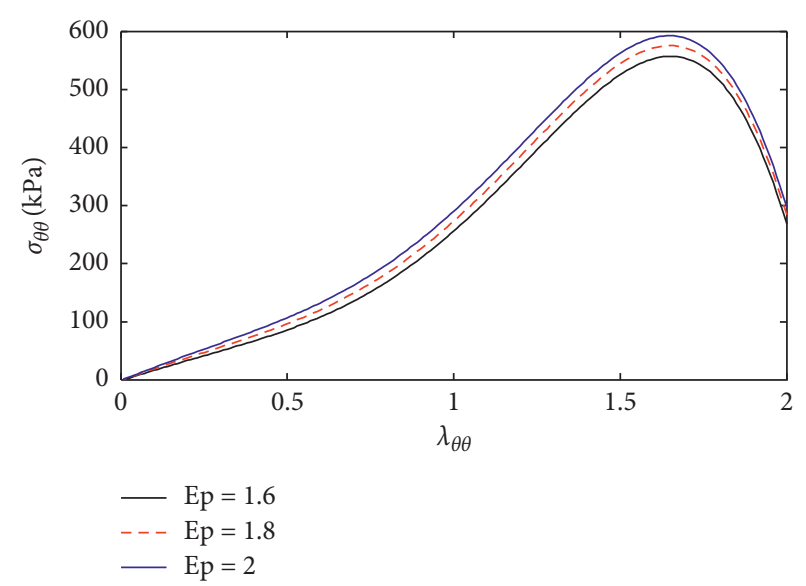

FIGURE 10: Study of the influence of the elasticity modulus on circumferential stress.

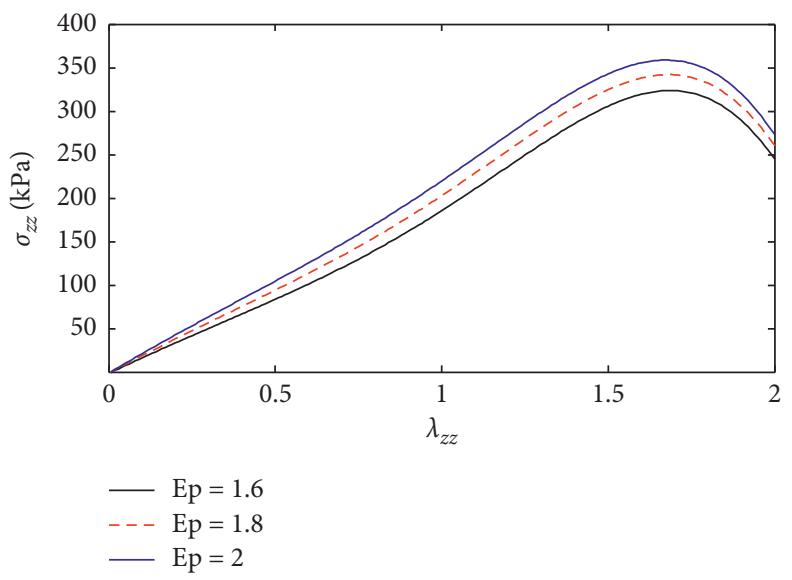

FIGURE 11: Study of the influence of the elasticity modulus on axial stress.

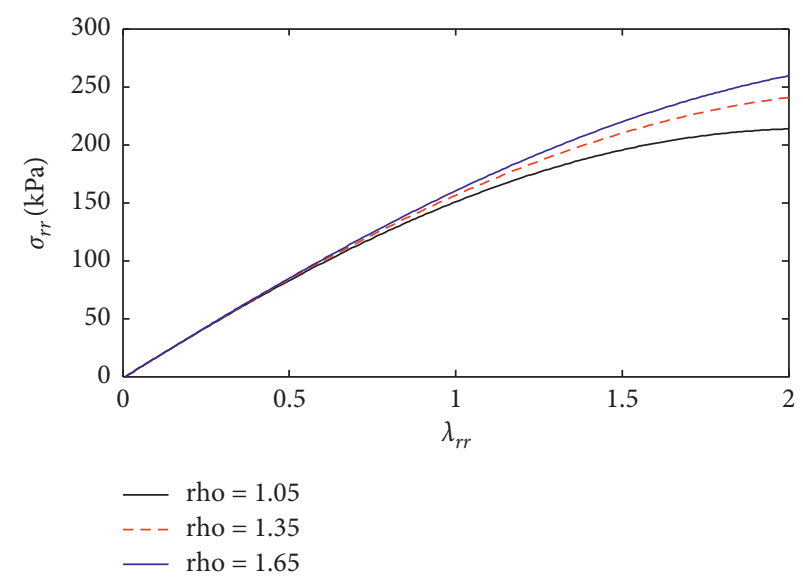

FIgURE 12: Influence of blood density on radial stress. 


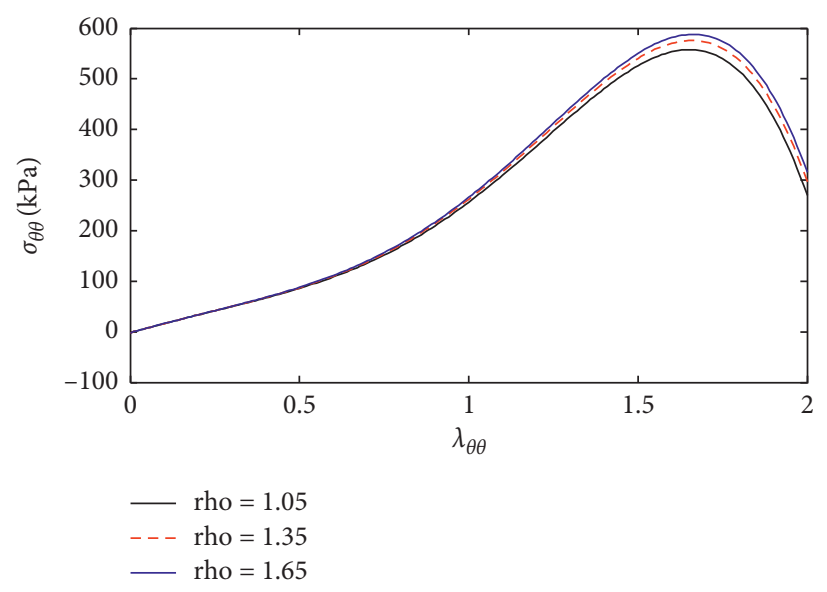

FIGURE 13: Influence of blood density on circumferential stress.

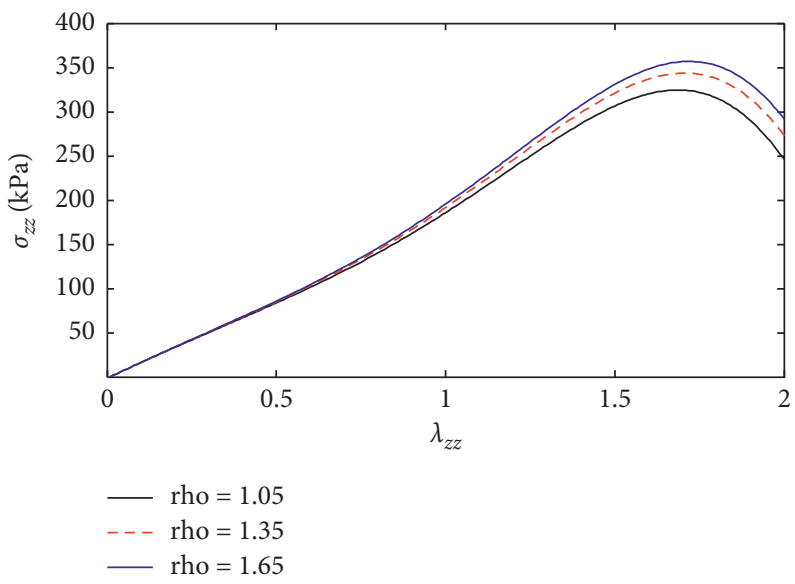

FIGURE 14: Influence of blood density on axial stress.

3.3. Study of the Influence of the Elasticity Modulus (EP). Figures 9-11 show the influence of the elasticity modulus on the resistance of the arterial wall. It is obvious that when the elasticity modulus increases $(\mathrm{EP})=1.6 ; 1.8 ; 2)$, the stress also increases. The rise of the latter (EP) leads to the loss of elasticity of the arterial wall [43]. This loss of elasticity is caused by arteriosclerosis. How is it possible?

Firstly, arteriosclerosis is characterized by a thickening, hardening, and loss of elasticity of the artery wall. It is a form of sclerosis that occurs in arteries. It is often defined as a natural, age-related phenomenon with normal thickening of the artery wall. However, many studies have also shown that this hardening of the wall can be accelerated by certain cardiovascular disorders. That is to say, the progressive deposition of lipids in the artery walls which causes the artery walls to thicken and harden [44]. In this case, one speaks more often of atherosclerosis in reference to atheroma, which refers to the plaque of fat formed. In addition, sclerosis of the arteries can be favoured by many factors such as genetic factors, metabolic disorders, poor eating habits, lack of physical activity, and certain stress factors. Finally, arteriosclerosis can remain asymptomatic for several years.
Nevertheless, in the most serious cases, it can block arteries that are essential to the proper functioning of the body, such as the coronary and carotid arteries. It leads to poor oxygenation and wall stiffness [45]. The prevention of arteriosclerosis consists in reducing the modulus of elasticity of the wall by limiting risk factors such as bad eating habits and sedentary lifestyle. To achieve, it is recommended to adopt a healthy and balanced diet by limiting the consumption of processed products and excess fat, sugar, and alcohol and by regular physical activity.

3.4. Study of the Influence of the Blood Density Mass. Figures 12-14 present the influence of the density of the blood on the stress of the arterial wall. It is evident that an increase in the latter increases the stress on the arterial wall [46]. This is due to the fact that when the arterial lumen is reduced, the particle of blood (hematocrit...) is unable to move well, see Figure 15.

Thus, thrombosis joins the blood platelets and causes an increase in the density of the blood and the particle of blood is unable to move well [47]. For a longtime, this can cause blood clot and can lead to the destruction of the artery wall. 


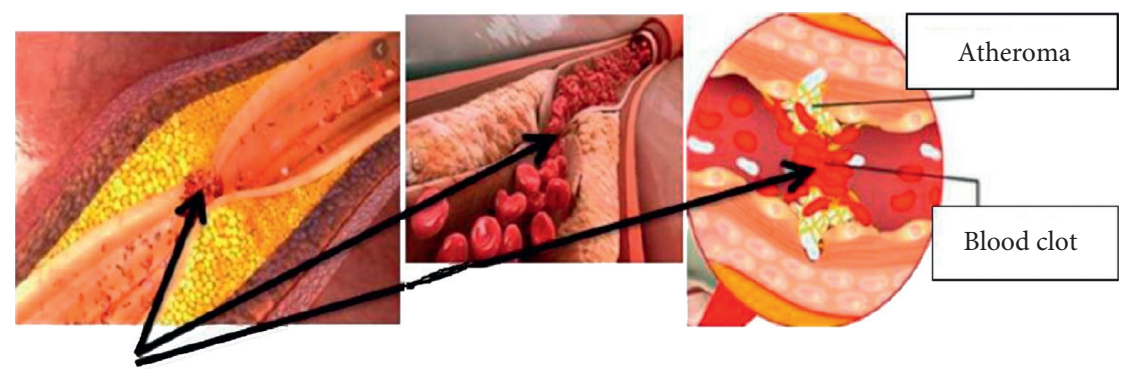

FIgURE 15: Blood density illustration (health on the net.com).

\section{Conclusion}

In this article, we studied the distribution of arterial stress, taking into account the properties of the hydrostatic and the evolution of the stenosis. We have coupled these two characteristics to the model of hyperelastic, anisotropic, and dynamic behavior of the human artery. This improvement of the model is useful for understanding the radial and longitudinal growth of plaque on arterial stress. The effects of the blood density mass, of the elasticity module, and of the atherosclerosis's growth have been deeply examined. The model allowed us to show that the radial growth of the plaque is very dangerous compared to the longitudinal growth. The plaque also promotes increased density and loss of elasticity. All this new knowledge will help us predict arterial damage and could also help the doctor and the surgeon to make a good clinical diagnosis, decisions, and good treatment planning.

\section{Data Availability}

The data used in this paper are from papers cited in the reference and in the text.

\section{Conflicts of Interest}

The authors declare that they have no conflicts of interest.

\section{References}

[1] R. Singh, K. P. Singh, S. Cherian et al., "Rabies-epidemiology, pathogenesis, public health concerns and advances in diagnosis and control: a comprehensive review," Veterinary Quarterly, vol. 37, no. 1, pp. 212-251, 2017.

[2] S.-s. Zhou, J.-p. Jin, J.-q. Wang et al., "miRNAS in cardiovascular diseases: potential biomarkers, therapeutic targets and challenges," Acta Pharmacologica Sinica, vol. 39, no. 7, pp. 1073-1084, 2018.

[3] R. Ponalagusamy, "A two-layered suspension (particle-fluid) model for non-Newtonian fluid flow in a catheterized arterial stenosis with slip condition at the wall of stenosed artery," Korea-Australia Rheology Journal, vol. 29, no. 2, pp. 87-100, 2017.

[4] A. Akter, "Mathematical analysis of a non-Newtonian model for blood flow considering shear rate dependent viscosity through a stenosed artery in presence of magnetic field," 2019, http://lib.buet.ac.bd:8080/xmlui/handle/123456789/5412.

[5] A. Saxena, E. Y. K. Ng, M. Mathur, C. Manchanda, and N. A. Jajal, "Effect of carotid artery stenosis on neck skin tissue heat transfer," International Journal of Thermal Sciences, vol. 145, Article ID 106010, 2019.

[6] J. Ding, J. Guan, X. Ji, and R. Meng, "Cerebral venous sinus stenosis may cause intracranial arterial hypoperfusion," Clinical Neuroradiology, vol. 30, no. 2, pp. 409-411, 2019.

[7] K. K. Wong, J. Wu, G. Liu, W. Huang, and D. N. Ghista, "Coronary arteries hemodynamics: effect of arterial geometry on hemodynamic parameters causing atherosclerosis," Medical \& Biological Engineering \& Computing, vol. 58, pp. 1831-1843, 2020.

[8] L. de Figueiredo Borges, R. G. Jaldin, R. R. Dias, N. A. G. Stolf, J.-B. Michel, and P. S. Gutierrez, "Collagen is reduced and disrupted in human aneurysms and dissections of ascending aorta," Human Pathology, vol. 39, no. 3, pp. 437-443, 2008.

[9] N. F. MacLean, N. L. Dudek, and M. R. Roach, "The role of radial elastic properties in the development of aortic dissections," Journal of Vascular surgery, vol. 29, no. 4, pp. 703-710, 1999.

[10] A. Danpinid, J. Luo, J. Vappou, P. Terdtoon, and E. E. Konofagou, "In vivo characterization of the aortic wall stress-strain relationship," Ultrasonics, vol. 50, no. 7 , pp. 654-665, 2010.

[11] K. Khanafer and R. Berguer, "Fluid-structure interaction analysis of turbulent pulsatile flow with in a layered aortic wall as related to aortic dissection," Journal of Biomechanics, vol. 42, pp. 2642-2648, 2009.

[12] C. J. Lee, Y. Zhang, H. Takao, Y. Murayama, and Y. Qian, “A fluid-structure interaction study using patient-specific ruptured and unruptured aneurysm: the effect of aneurysm morphology, hypertension and elasticity," Journal of Biomechanics, vol. 46, no. 14, pp. 2402-2410, 2013.

[13] S. Dhar, M. Tremmel, J. Mocco et al., "Morphology parameters for intracranial aneurysm rupture risk assessment," Neurosurgery, vol. 63, no. 2, pp. 185-197, 2008.

[14] Ø. Evju, K. Valen-Sendstad, and K.-A. Mardal, "A study of wall shear stress in 12 aneurysms with respect to different viscosity models and flow conditions," Journal of Biomechanics, vol. 46, no. 16, pp. 2802-2808, 2013.

[15] B. J. Doyle, J. Killion, and A. Callanan, "Use of the photoelastic method and finite element analysis in the assessment of wall strain in abdominal aortic aneurysm models," Journal of Biomechanics, vol. 45, no. 10, pp. 1759-1768, 2012.

[16] H. Masson, P. Beaussier, S. Boutouyrie, J. D. Laurent, and M. Humphrey, "Modélisation du comportement mécanique d'artères carotides communes humaines in vivo," in Proceedings of the Congrès Français de Mécanique, pp. 24-28, Marseille, France, 2009.

[17] G. Holzapfel, Nonlinear Solid Mechanics: A Continuum Approach for Engineering, John Wiley \& Sons Inc, New York, NY, USA, 2000. 
[18] T. E. Carew, R. N. Vaishnav, and D. J. Patel, "Compressibility of the arterial wall," Circulation Research, vol. 23, no. 1, pp. 61-68, 1968.

[19] T. C. Gasser, G. Görgülü, M. Folkesson, and J. Swedenborg, "Failure properties of intraluminal thrombus in abdominal aortic aneurysm under static and pulsating mechanical loads," Journal of Vascular Surgery, vol. 48, no. 1, pp. 179-188, 2008.

[20] H. Demiray and R. P. Vito, "A layered cylindrical shell model for an aorta," International Journal of Engineering Science, vol. 29, no. 1, pp. 47-54, 1991.

[21] G. A. Holzapfel, M. Stadler, and T. C. Gasser, "Changes in the mechanical environment of stenotic arteries during interaction with stents: computational assessment of parametric stent designs," Journal of Biomechanical Engineering, vol. 127, no. 1, pp. 166-180, 2005.

[22] F. Cacho, M. Doblaré, and G. A. Holzapfel, "A procedure to simulate coronary artery bypass graft surgery," Medical \& Biological Engineering \& Computing, vol. 45, no. 9, pp. 819827, 2007.

[23] V. Alastrué, M. A. Martínez, and M. Doblaré, "Modelling adaptative volumetric finite growth in patient-specific residually stressed arteries," Journal of Biomechanics, vol. 41, no. 8, pp. 1773-1781, 2008.

[24] J. Merodio and R. W. Ogden, "On tensile instabilities and ellipticity loss in fiber-reinforced incompressible non-linearly elastic solids," Mechanics Research Communications, vol. 32, no. 3, pp. 290-299, 2005.

[25] S. Jonas, "A lumped parameter model of cerebral blood flow regulation: applications to simulation of carotid endarterectomy," Master's thesis, Technische Universität München, Munich, Germany, 2015.

[26] B. Spronck, E. G. Martens, E. D. Gommer, and F. N. van de Vosse, "A lumped parameter model of cerebral blood flow control combining cerebral autoregulation and neurovascular coupling," American Journal of Physiology. Heart and Circulatory Physiology, vol. 303, pp. H1143-H1153, 2012.

[27] R. Aaslid, "Visually evoked dynamic blood flow response of the human cerebral circulation," Stroke, vol. 18, no. 4, pp. 771-775, 1987.

[28] R. Chadwick, "Mechanics of the left ventricle," Biophysical Journal, vol. 39, no. 3, p. 27988, 1982.

[29] H. Cai, "Loi de comportement en grandes déformations du muscle à fibres actives: application à la mécanique du coeur humain et à sa croissance," Doctoral thesis, Université de Savoie, Chambéry, France, 1988.

[30] J. D. Humphrey, R. K. Strumpf, and F. C. P. Yin, "Determination of a constitutive relation for passive myocardium: II-parameter estimation," Journal of Biomechanical Engineering, vol. 112, no. 3, pp. 340-346, 1990.

[31] M. P. Nash and P. J. Hunter, "Computational mechanics of the heart," Journal of Elasticity, vol. 61, no. 1-3, pp. 113-141, 2000.

[32] J. D. Humphrey and S. Na, "Elastodynamics and arterial wall stress," Annals of Biomedical Engineering, vol. 30, no. 4, pp. 509-523, 2002.

[33] A. Valentín, L. Cardamone, S. Baek, and J. D. Humphrey, "Complementary vasoactivity and matrix remodelling in arterial adaptations to altered flow and pressure," Journal of the Royal Society Interface, vol. 6, no. 32, pp. 293-306, 2009.

[34] J. Alastruey, K. Parker, J. Peiró, S. Byrd, and S. Sherwin, "Modelling the circle of willis to assess the effects of anatomical variations and occlusions on cerebral flows," Journal of Biomechanics, vol. 40, no. 8, pp. 1794-1805, 2007.
[35] G. Mulder, A. Bogaerds, P. Rongen, and F. van de Vosse, "The influence of contrast agent injection on physiological flow in the circle of Willis," Medical Engineering \& Physics, vol. 33, pp. 195-203, 2011.

[36] M. Ursino and C. A. Lodi, "Interaction among autoregulation, $\mathrm{CO}_{2}$ reactivity, and intracranial pressure: a mathematical model," American Journal of Physiology-Heart and Circulatory Physiology, vol. 274, no. 5, pp. H1715-H1728, 1998.

[37] S. M. Moore, "Computational 3D modelling of hemodynamics in the circle of Willis," Ph.D. thesis, University of Canterbury, Christchurch, New Zealand, 2007.

[38] O. Kurihara, M. Takano, T. Soeda et al., "Degree of luminal narrowing and composition of thrombus in plaque erosion," Journal of Thrombosis and Thrombolysis, vol. 51, pp. 143-150, 2020.

[39] J. T. Tsafack, J. M. Takam, M. Fogue, and P. K. Talla, "Study of cerebral blood flow by the lumped parameter model to predict the rupture of the arterial wall: application to stroke," International Journal of Academic Research and Reflectionvol, vol. 7, no. 6, pp. 2309-0405, 2019.

[40] R. Pandey, M. Kumar, J. Majdoubi, M. Rahimi-Gorji, and V. K. Srivastav, "A review study on blood in human coronary artery: numerical approach," Computer Methods and Programs in Biomedicine, vol. 187, Article ID 105243, 2020.

[41] J. M. López, G. Fortuny, D. Puigjaner, J. Herrero, and F. Marimon, "Hemodynamic effects of blood clots trapped by an inferior vena cava filter," International Journal for $\mathrm{Nu}$ merical Methods in Biomedical Engineering, vol. 36, p. e3343, 2020.

[42] M. Biglarian, M. M. Larimi, H. H. Afrouzi et al., "Computational investigation of stenosis in curvature of coronary artery within both dynamic and static models," Computer Methods and Programs in Biomedicine, vol. 185, Article ID 105170, 2020.

[43] K. Xu, L. Yu, J. Wan, S. Wang, and H. Lu, "The influence of the elastic modulus of the plaque in carotid artery on the computed results of FFRCT," Computer Methods in Biomechanics and Biomedical Engineering, vol. 23, no. 5, pp. 201-211, 2020.

[44] A. Tajbakhsh, P. T. Kovanen, M. Rezaee, M. Banach, S. A. Moallem, and A. Sahebkar, "Regulation of efferocytosis by caspase-dependent apoptotic cell death in atherosclerosis," The International Journal of Biochemistry \& Cell Biology, vol. 120, Article ID 105684, 2020.

[45] J. Barallobre-Barreiro, B. Loeys, M. Mayr, M. Rienks, A. Verstraeten, and J. C. Kovacic, "Extracellular matrix in vascular disease, part 2/4," Journal of the American College of Cardiology, vol. 75, no. 17, pp. 2189-2203, 2020.

[46] P. Mythravaruni and K. Y. Volokh, "Residual stresses reduce pulse wave velocity in arteries," Mechanics of Soft Materials, vol. 2, p. 7, 2020.

[47] M. Al-Saad, C. A. Suarez, A. Obeidat, S. P. A. Bordas, and S. Kulasegaram, "Application of smooth particle hydrodynamics method for modelling blood flow with thrombus formation," Computer Modeling in Engineering \& Sciences, vol. 122, no. 3, pp. 831-862, 2020. 